\title{
Index rerum ad Vol. 150
}

\section{Confecit F. Ammann, Geneve}

(B) = Buchbesprechungen $-\mathrm{Ll} \cdot$ vres nouveaux - Book reviews. $(\mathrm{R})=$ Referate von Vorträgen, die an anderer Stelle ausfûhrlich erschienen sincl oder erscheinen werden. Einzelheiten werden in diesem Index rerum nicht beriicksichtigt. - Rapports de conferences qui ont été (ou seront) publiées ailleurs in extenso. Cet Index rerum ne contient pas de details. - Abstract $\mathrm{s}$ of lectures that have been (or will be) published elsewhere. Details are not included in this Index rerum.

Abcès annulaire, v. Ringabszeß Acanthocytosis-like erythrocytes,

v. Eales' disease Ätiologie der ischämischen Papillenschwellung, 241 Age; influence of age on enzyme activities of lenses, 187 Aging eye, 157 (B) Albright; la cataracte et $\Gamma$ ostéodystrophie héréditaire d'Albright, 409 Altersbedingte Veränderungen, v. Lenses Alupent; zur Frage der Augeninnendruckbeeinflussung durch Alupent, Silomat und deren Kombination, 252 Amblyopie; à propos de Tamblyopie à fixation excentrique, 76 Amotio retinae, v. Netzhautablösung, Retinal detachment, Rißamotio Anaphylactic uveitis, v. Uveitis, experimental Applanation tonography, 65 Applanationstonometrie, v. Tonometrie Arteriitis temporalis, v. Papillen-

schwellung Asteroid hyalitis, v. Scintillatio nivea Atlas der Augenkrankheiten, 92 (B) Augenbinnendruck, v. Alupent, Glaucome phakolytique, Glaucome simple, Hydrophthalmos, Scleral rigidity, Tonography, Tonometrie, Viadril Augenheilkunde; Unterrichtsbuch für das augenärztliche Hilfspersonal (3.Aufl.), 158 (B) Augenhintergrundsveränderungen, v. Chlorochinretinopathie, Eales' disease, Fundus xerophthalmicus, Papillenschwellung Augeninnendruckbeeinflussung; zur Frage der Augeninnendruckbeeinflussung durch Alupent, Silomat und deren Kombination, 252

Augenkomplikationen, v. Alupent, Chlorochinretinopathie Axe antéro-postérieur; sur Гerreur de la mesure de Гaxe antéro-postérieur de $\Gamma æ i l$ au moyen d'ultra-sons dérivée de Tadoption d'une vitesse de propagation constante, 431 Basaliome; zur Klinik und Therapie der Basaliome und Spinaliome, 138 Betamethason, v. Celestone Bibliographie, v. Manuskript Bindehaut, v. Keratoconjunctivitis epidemica Biometrie; biometrische Befunde bei 
Rißamotio im kurzsichtigen Auge.

Beitrag zur Pathogenese, 386 Biometrie, v. Axe antéro-postérieur Biomikroskopische Untersuchungen

nach Fluoreszeininjektion, 371 Blindheit, v. Blindness Blindness; mother and blind child, 240 (B) Bceuf, v. Lenses Book reviews, 92 (B), 157-159 (B),

239-240 (B),318 (B) Bovine lenses, v. Lenses Buchbesprechungen, 92 (B), 157-159

(B), 239-240 (B),318 (B)

Carotis-Thrombose, v. Papillenschwellung

Cataract absorption; spontaneous cataract absorption in Haller-mann-Streiff syndrome, 401

462

Index rerum

ad Vol. 150

Cataract, senile; further investigations on some properties of the blood of patients with senile cataract, 196

Cataracte; la cataracte et Tostéodys-trophie héréditaire d'Albright, 409

Cataracte, v. Pseudo-exfoliation capsulaire

Cécité, v. Blindness

Celestone; Erfahrungen mit Beta-methason (Celestone) bei der Be-handlung entzündlicher

Affektio-nen des Auges, 263

Champ visuel, v. Cortisone, Visual fields

Chemotherapeutica et Antibiotica, v. Celestone, Cytostatique, Viadril

Chicken lenses, v. Lenses

Chirurgie plastique, v. Operationen, wiederherstellende

Chlorochinretinopathie; die Morpho-logie der experimentellen Chlorochinretinopathie d.

Kaninchens, 127

Cobaye, v. Lenses

Coefficient de rigidité, v. Tonometrie

Complications oculaires, v. Alupent, Chlorochinretinopathie

Congrès, annonce de, v. Congrès d'Ophtalmologie, Varia

Congrès d'Ophtalmologie; communications relatives au XXe Congrès International

d'Ophtalmologie (Munich, 14-19 août 1966), 319

Congress, v. Ophthalmological Society of the United Kingdom

Congress of Ophthalmology; information of the XXth International Congress of Ophthalmology

(Munich, August 14-19, 1966), 93

Conjunctiva, v. Keratoconjunctivitis epidemica

Conjunctivitis, v. Celestone

Contact lenses, v. Keratoconus

Cornea, v. Histiocytome, Ring-abszess, Secretion lacrymaîe

Corneal preservation, v. Hornhaut-aufbewahrung

Corps vitré, v. Scintillatio nivea

Corticosteroid, v. Celestone

Cortisone; le test à la cortisone dans le glaucome simple à champ visuel normal, 46

Cristallin, v. Cataract absorption, Cataract, senile, Cataracte, Glaucome phakolytique, Lenses,

Lens proteins, Lenticonus, Pseudo-exfoliation capsulaire

Cryptophtalmie; syndrome malfor-matif avec cryptophtalmie (note préliminaire), 215 
Cuppers, méthode de, v. Amblyopie

Cytostatique; influence d'un nou-veau cytostatique (derive de la méthylhydrazine) sur $\Gamma$ uvéite ex-périmentale du lapin, 97

Déchirure de la rétine, v. Rißamotio Décollement rétinien, v. Netzhaut-

ablösung, Retinal detachment,

Rißamotio Detachment of retina, v. Netzhaut-

ablösung, Retinal detachment,

Rißamotio Diagnosis, v. Glaucome simple Diagnostik maligner Tumoren am

hinteren Augenpol mittels P32, 153 Diaphanoskopie; Intensiv-Diapha-

noskopie, 441 Diplopien; eine objektive Methode

zur Analyse der Diplopien, 347 Documenta Ophthalmologica

(Vol. 18), 157 (B) Doppelbilder, v. Diplopien

Eales' disease; lymphoreticular reactions in Eales' disease, 270

Echographie, v. Axe antéro-posté-rieur, Myopie

Electro-oculogram; the influence of changes in illumination on the standing potential of the

human eye, 83

Electroretinography; physiology of flicker/flicker electroretinography (Documenta

Ophthalmologica, Vol. 18), 157 (B)

EOG, v. Electro-oculogram

ERG, v. Electroret...

Experimentelle Forschung, v. Cytostatique, Lenses, Lens proteins, Mißbildungsrosetten, Netzhaut-ablösung, Viadril

Fixation excentrique, v. Amblyopie Flicker, v. Electroretinography

Index rerunt

ad Vol. 150

463

Fluorescein angiography; diabetic retinopathy studied by fluorescein angiography, 5

Fluoreszeininjektion; biomikrosko-pische Untersuchungen nach Fluoreszeininjektion, 371

Fortbildung und Orientierung, 153

Fuchs, excavations marginales de la cornée de, v. Secretion lacrymale

Fundus, v. Chlorochinretinopathie, Eales' disease, Papillenschwellung

Fundus xeГophthalmicus; further contributions to the fundus xerophthalmicus, 219

Further studies and other information, 153

Gefäße, v. Eales' disease, Fluorescein angiography, Microvascular methodology,

Papillenschwellung

Génétique ophtalmologique,

v. Cryptophtalmie, Humangenetik, Lenticonus, Mikrophthalmus, Ostéodystrophie héréditaire

Gerontology, v. Aging eye, Cataract, senile

Gesichtsfeld, v. Cortisone, Visual fields

Glaskörper, v. Scintillatio nivea

Glaucoma, v. Alupent, Hydrophthal-mos, Iridenkleisis, Pseudo-exfoliation capsulaíre

Glaucome phakolytique, 36

Glaucome simple; le test à la cortisone dans le glaucome simple a champ visuel normal, 46

Graft, corneal, v. Hornhautaufbe-wahrung, Keratoconus, Operatio-nen, wiederherstellende

Greffe cornéenne, v. Hornhautauf-bewahrung, Keratoconus, Opera-tionen, wiederherstellende 
Guinea pig lenses, v. Lenses

Hähnchenlinsen, v. Lenses

Hallermann-Streiff; spontaneous cataract absorption in Hallermann-Streiff syndrome, 401

Hémorragies récidivantes des jeunes gens, v. Eales' disease

Heredität, v. Cryptopthalmie, Humangenetik, Lenticonus, Mikrophthalmus, Ostéodystrophie

héréditaire

Histiocytome; à propos de Thistio-cytome de la cornée à evolution fibromateuse, 306

Histochemie, v. Keratoconjunctivitis epidemica, Lenses, Lens proteins

Histologie, v. Chlorochinretinopathie, Hallermann-Streiff, Histiocytome, Mißbildungsrosetten

Hornhaut, v. Histiocytome, Ring-abszeß, Secretion lacrymale

Hornhautaufbewahrung; Aufbewah-rung von Hornhaut in wasser-freiem Glyzerin, 155

Hornhautplastik, v. Hornhautaufbewahrung, Keratoconus, Opera-tionen, wiederherstellende

Hruby lens, v. Fluorescein angiography

Humangenetik, 92 (B)

Hydrophthalmos; a contribution to the surgical treatment of hydrophthalmos, 57

Hydroxydione sodium succinate, v. Viadril

Hypertension oculaire, v. Alupent, Glaucome phakolytique, Glaucome simple, Hydrophthalmos,

Scleral rigidity, Tonography, Tonometrie

Hypotension, ocular, v. Viadril

Industrial and traumatic ophthalmology (Symposium of the New Orleans Academy of

Ophthalmology), 159 (B) Inflammations, v. Celestone Instrument, v. Diaphanoskopie Intraocular pressure, v. Alupent, Glaucome phakolytique, Glaucome simple, Hydrophthalmos, Scleral rigidity, Tonography, Tonometrie, Viadril Intraokularer Druck, v. Alupent, Glaucome phakolytique, Glaucome simple, Hydrophthalmos, Scleral rigidity, Tonography, Tonometrie, Viadril Iridenkleisis: Modifikationen und Resultate, 422

Kaninchen, v. Chlorochinretinopathie, Lens proteins, Uvéite ex-périmentale, Viadril

464

Index rerum

ad Vol. 150

Katarakt, v. Cataract absorption, Cataract, senile, Cataracte, Pseudo-exfoliation capsulaire Keratitis, v. Celestone

Keratoconjunctivitis epidemica; Enzymwirkungen und cytologi-sche Veränderungen bei

Keratoconjunctivitis epidemica, 292

Keratoconus; indications of contact lenses or keratoplasty in keratoconus, 161

Keratoplastik, v. Hornhautaufbe-wahrung, Keratoconus, Operatio-nen, wiederherstellende

Kongreßkalender, v. Ophthalmolo-gen-Kongreß, Varia

Kontaktgläser, v. Keratoconus

Lacrymal secretion, v. Secretion

lacrymale Lapin, v. Chlorochinretinopathie,

Lens proteins, Uvéite expérimen-

tale, Viadril Lens, v. Cataract absorption,

Cataract, senile, Cataracte, Glau-

come phakolytique, Lenticonus,

Pseudo-exfoliation capsulaire Lenses; influence of age on enzyme

activities of lenses, 187 Lens proteins; early modifications 
of the soluble lens proteins in

the course of experimental uveitis,

452 Lenticonus; bilateral anterior and

posterior lenticonus, 107 Libri, 92 (B), 157-159 (B),

239-240 (B), 318 (B) Lid, v. Basaliome, Spinaliome Linse, v. Cataract absorption,

Cataract, senile, Cataracte, Glau-

come phakolytique, Lenticonus,

Pseudo-exfoliation capsulaire Literaturverzeichnis, v. Manuskript Livres nouveaux, 92 (B), 157159

(B), 239-240 (B),318 (B)

Maladie d'Eales, v. Bales' disease Malformations oculaires, v. Crypt-ophtalmie, Humangenetik, Lenticonus, Mißbildungsrosetten Malformative syndrome, v. Crypt-ophtalmie

Manuskript; das Manuskript, 159 (B)

Medical meetings, announcement of, v. Congress of Ophthalmology, Varia

Meerschweinchen, v. Lenses

Methode, v. Amblyopie, Diplopien, Fluorescein angiography, Glau-come simple,

Hornhautaufbewah-rung, Iridenkleisis, Tonography, Tonometrie

Méthylhydrazine, v. Cytostatique

Microvascular methodology,

symposium on (Acta Anatomica, Fasc. 5) , 239 (B)

Mikrophthalmus; über Form und Entwicklung von Mißbildungsrosetten beim hereditären

Mikrophthalmus der Hausmaus, 359

Mißbildungen des Auges, v. Crypt-ophtalmie, Humangenetik, Lenticonus, Mißbildungsrosetten

Mißbildungsrosetten; über Form und Entwicklung von Mißbildungsrosetten beim hereditären

Mikrophthalmus der Hausmaus, 359

Mouse, v. Mikrophthalmus

Myopie; biometrische Befunde bei Rißamotio im kurzsichtigen Auge. Beitrag zur Pathogenese, 386

Necroses cornéennes, v. Secretion lacrymale

Nervus opticus, v. Papillenschwel-lung

Netzhautablösung, 158 (B)

Netzhautablösung, v. Retinal detachment, Rißamotio

Netzhautrosetten, v. Mißbildungsrosetten

Ocular rigidity, v. Scleral rigidity Ocular tension, v. Alupent, Glau-

come phakolytique, Glaucome

simple, Hydrophthalmos, Scleral

rigidity, Tonography, Tonometrie,

Viadril Oculocardiac reflex during surgical

correction of strabismus in local

anesthesia, 259 Oedème de la papille, v. Papillen-

schwellung

Index $\Gamma$ erum

ad Vol. 150

465

Oftalmología; traumatología ocular y oftalmología laboral, 239 (B) 
-temas oftalmológicos, 318 (B)

Operation, v. Basaliome, Hydroph-thalmos, Iridenkleisis, Keratoco-nus, Netzhautablösung, Pterygium, Retinal detachment, Spinaliome

Operationen, wiederherstellende; wiederherstellende Operationen bei schweren Schädigungen und Erkrankungen des Auges, 239 (B)

Ophthalmologen-Kongreß; Mittei-lungen über den XX. Internationa-len OphthalmologenKongreß (München, 14. bis 19. August 1966), 95

Ophthalmologica; Documenta Oph-thalmologica (Vol. 18), 157 (B)

Ophthalmological Society of the United Kingdom, 85th congress (1965), 457-460 (R)

Ophthalmologie, v. Augenheilkunde

Ophthalmology; questions and answers in ophthalmology, 158 (B)

-; industrial and traumatic ophthalmology (Symposium of the New Orleans Academy of

Ophthalmology), 159 (B)

Ophthalmology, year book (1963-1964), 318 (B)

Optics and the eye, 157 (B)

Optikomalazie, v. Papillenschwel-lung

Ostéodystrophie héréditaire; la cataracte et Гostéodystrophie héréditaire d'Albright, 409

Oto-Neuro-Ophthalmologie, Bericht der Tagung über, 318 (B)

Papilledema, v. Papillenschwellung Papillenschwellung; zur Ätiologie der ischämischen

Papillenschwellung, 241 Pathogenesis, v. Eales' disease, Papillenschwellung, Rißamotio,

Scintillatio nivea, Secretion lacrymale Paupière, v. Basaliome, Spinaliome Perfectionnement et orientation, 153 Perimetry, v. Visual fields Periphlebitis, retinal, v. Eales' disease

Phacoanaphylactic endophthalmitis, v. Hallermann-Streiff

Phacolytic glaucoma, v. Glaucome phakolytique

Physiologie, v. Axe antéro-posté-rieur, Electro-oculogram, Electro-retinography

Plastic surgery, v. Operationen, wiederherstellende

Potentiel de repos, v. Standing potential

Poulet, v. Lenses

Pression oculaire, v. Alupent, Glaucome phakolytique, Glaucome simple, Hydrophthalmos,

Scleral rigidity, Tonography, Tonometrie, Viadril

Proceedings, 457-460 (R)

Protein-SH groups, v. Cataract, senile, Lens proteins

Pseudo-exfoliation capsulaire, 175

Pseudo-hypoparathyroïdisme, v. Albright

Pseudomonas pyocyanea, v. Ringabszeß

Ptérygion, v. Secretion lacrymale

Pterygium; a new method of

operation giving more successful results in pterygium disease, 123

Pterygium throughout the world, 240 (B)

Pyocyaneusinfektion, v. Ringabszeß

Rabbit, v. Chlorochinretinopathie, Lens proteins, Uvéite expérimen-tale, Viadril

Recherches expérimentales, v. Cyto-statique, Lenses, Lens proteins, Mißbildungsrosetten,

Netzhautablösung, Viadril

Retina, v. Mißbildungsrosetten

Retinal detachment; results and remarks on some recent techniques employed in the treatment of retinal detachment (with especial emphasis on encircling methods), 18 
Retinal detachment, v. Netzhautablösung, Rißamotio

Retinopathy, diabetic, v. Fluorescein angiography

Rheology, v. Microvascular methodology

466

Index rerunı

ad Vol. 150

Rheology of the human sclera.

Unifying formulation of ocular

rigidity, 321 Rigiditätskoeffizient, v. Tonometrie Rigidity, v. Scleral rigidity Rinderlinsen, v.

Lenses Ringabszeß; zur Therapie des Ring-

abszesses der Hornhaut nach In-

fektion mit Pseudomonas pyo-

cyanea, 353 Rißamotio; biometrische Befunde

bei Rißamotio im kurzsichtigen

Auge. Beitrag zur Pathogenese,

386 Ro 4-6467, v. Cytostatique Rosetten, v. Mißbildungsrosetten Ruhepotential, v. Standing potential

Schielen, v. Amblyopie, Diplopien

Schieloperation, v. Oculocardiac reflex

Schiøtz-Tonometer, v. Tonometrie

Scintillatio nivea; ätiologische Be-trachtungen zur Scintillatio nivea, 167

Scleral rigidity; rheology of the human sclera. Unifying formulation of ocular rigidity, 321

Secretion lacrymale; la discontinuite du film lacrymal précornéen - cause des excavations

marginales de la cornée de Fuchs, de la progression du ptérygion et de cer-taines necroses de la cornée au voisinage des kératoprothèses et kératoplasties, Ill

Sehschwäche, v. Amblyopie

Senescence, v. Aging eye, Cataract, senile

Silomat-Bronchial-Elixier, v. Alupent

Sitzungsberichte, $457-460$ (R)

Society transactions, $457-460$ (R)

Souris, v. Mikrophthalmus

Spinaliome; zur Klinik und Therapie der Basaliome und Spinaliome, 138

Squint, v. Amblyopie, Diplopien

Squintoperation, v. Oculocardiac reflex

Standing potential; the influence of changes in illumination on the standing potential of the human eye, 83

Star, grauer, v. Cataract absorption, Cataract, senile Cataracte Pseudo-exfoliation capsulaire

Statistische Auswertung, v. Amblyopie, Basaliome, Fundus xeroph-thalmicus, Iridenkleisis,

Pseudo-exfoliation capsulaire, Retinal detachment, Spinaliome

Strabismus; oculocardiac reflex during surgical correction of strabismus in local anesthesia, 259

Strabismus, v. Amblyopie, Diplopien

Sulfhydryl groups, v. Cataract, senile, Lens proteins

Sympathicomimeticum, v. Alupent

Syndrome, v. Albright, Hallermann-Streiff

Syndrome malformatif avec cryptophtalmie (note prélimi-naire), 215 
Target cells, v. Eales' disease

Tear, retinal, v. Rißamotio

Tension oculaire, v. Pression oculaire

Test à la cortisone dans le glaucome simple à champ visuel normal, 46

Therapie, v. Amblyopie, Basaliome, Celestone, Keratoconus, Netzhaut-ablösung, Ringabszeß,

Spinaliome

Tonography; on applanation tono-graphy, 65

Tonometrie; zur Statistik der Tonometrie, 203

Tränensekretion, v. Secretion lacrymale

Transillumination, v. Diaphano-skopie

Transplantation, v. Hornhautauf-bewahrung, Keratoconus, Opera-tionen, wiederherstellende

Traumatic ophthalmology; industrial and traumatic ophthalmology (Symposium of the New

Orleans Academy of Ophthalmology), 159 (B)

Traumatología ocular y oftalmología laboral, 239 (B)

Tumor, v. Basaliome, Histiocytome, Spinaliome

Tumoren; die Diagnostik maligner Tumoren am hinteren Augenpol mittels P32, 153

Index rerum ad Vol. 150

467

Ultraschall, v. Axe antéro-postérieur, Myopie

Ultrasons, v. Axe antéro-postérieur, Myopie

Ultrasounds, v. Axe antéro-postérieur, Myopie

United Kingdom, v. Ophthalmo-logical Society

Untersuchungstechnik, v. Diapha-noskopie, Electro-oculogram, Electroretinography, Fluorescein angiography, Fluoreszeininjek-tion, Tonography, Tonometrie

Uvéite expérimentale; influence d'un nouveau cytostatique (derive de la méthylhydrazine) sur

Tuvéite expérimentale du lapin, 97

Uveitis, v. Celestone

Uveitis, experimental; early modifications of the soluble lens proteins in the course of

experimental uveitis, 452

Vaisseaux, v. Eales' disease,

Fluorescein angiography, Micro-

vascular methodology, Papillen-

schwellung Varia, 159, 240, 318 Vererbung, v. Cryptophtalmie, Hu-

mangenetik, Lenticonus, Mikroph-

thalmus, Ostéodystrophie héré-

ditaire Verhandlungsberichte, 457-460 (R) Verres de contact, v. Keratoconus Vessels, v. Eales'

disease, Fluorescein

angiography, Microvascular

methodology, Papillenschwellung Viadril; influence of Viadril on the

intraocular pressure of rabbits,

285 Visual field, v. Cortisone Visual fields (2nded.), 158 (B) Vitamin A deficiency, v. Fundus

xerophthalmicus Vitreous body, v. Scintillatio nivea

Xerophthalmia, v. Fundus xerophthalmicus

Year book of ophthalmology (1963-1964), 318 (B)

Index autorum ad Vol. 150 
Confecit F. Ammann, Geneve

(B) = Buchbesprechungen - Livres nouveaux - Book reviews. $(\mathrm{R})=$ Referate von Vorträgen, die an anderer Stelle ausführlich erschienen sind oder erscheinen werden. Die Autorennamen

wurden in diesem Index autorum nicht aufgenommen. - Rapports de conferences qui ont été (ou seront) publiées ailleurs in extenso. Cet Index autorum ne contient pas les noms des auteurs. Abstracts of lectures that have been (or will be) published in extenso elsewhere. The names of the authors have not been included in this Index autorum.

Auricchio, G., 196, 452

Barraquer, M. J. I., Ill

Batra, D. V., 107

Becker, P. E. (Editor), 92 (B)

Bellmann, O., 138

Benck, P., 46

Benkö, S., 270

B. Bettelheim

$\mathrm{H}$.

241 Bietti

G. B.

18 Bocci

$\mathrm{N}$.

196

452 Branemark

P. I.

239 (B) Burau

J.

259

Caldeira

J. A. F.

285 Cameron

M. E.

240 (B) 